\title{
Identifying indicators for quality abortion care: a systematic literature review
}

\author{
Amanda Dennis, ${ }^{1}$ Kelly Blanchard, ${ }^{1}$ Tshego Bessenaar ${ }^{2}$
}

${ }^{1}$ bis Reproductive Health, Cambridge, Massachusetts, USA ${ }^{2}$ bis Reproductive Health, Johannesburg, South Africa

\section{Correspondence to}

Tshego Bessenaar, Postnet Suite \# 197, Private Bag X2600, Houghton, Johannesburg 2041, South Africa; tbessanaar@ ibisreproductivehealth.org

Received 15 December 2015 Revised 9 March 2016 Accepted 7 April 2016 Published Online First 12 May 2016

\section{SLinked}

- http://dx.doi.org/10.1136/ jprhc-2016-101580

\section{CrossMark}

To cite: Dennis $A$, Blanchard K, Bessenaar T. J Fam Plann Reprod Health Care 2017:43:7-15.

\begin{abstract}
A systematic review was conducted of 13 peerreviewed articles and eight reports focused on indicators of quality abortion care. A total of 75 indicators of quality abortion were identified; these indicators address a variety of issues including policy, health systems, trained-provider availability, women's decision making, and morbidity and mortality. There is little agreement about indicators for measuring quality abortion care; more work is needed to ensure efforts to assess quality are informed and coordinated.
\end{abstract}

\section{BACKGROUND}

Both in well-resourced and underresourced health systems, it remains challenging to provide quality health services. This results in variation in the standards of health care delivery and in the outcomes of health care within and between health care systems. As it is a key component of ensuring equitable health care and outcomes, there is growing interest in improving the quality of health care across the globe. ${ }^{1}$

Despite its wide acceptance as an important health care issue, ${ }^{2}$ 'quality health care' remains variously and ambiguously defined. Historically, it has been defined primarily according to clinical standards. However, more recent definitions of health care quality are broad and multifaceted, accounting for the increasingly integrated nature of health care. ${ }^{3}$ For example, the Agency for Healthcare Research and Quality (AHRQ) in the USA defines quality health care to be "doing the right thing for the right patient, at the right time, in the right way to achieve the best possible results." 4 The Institute of Medicine in the USA, on the other hand, defines health care quality as "the extent to which health-care services improve health

\section{Key message points}

- A systematic review was conducted of 13 peer-reviewed articles and eight reports focused on indicators of quality abortion care.

- 75 indicators of quality abortion were identified; these indicators address a variety of issues including policy, health systems, trained-provider availability, women's decision-making, and morbidity and mortality.

- There is little agreement about indicators for measuring quality abortion care; more work is needed to ensure efforts to assess quality are informed and coordinated.

outcomes in a manner that is consistent with current professional knowledge." 5 Finally, the World Health Organization offers a more specific working definition, defining health care quality as health care that is "acceptable, accessible, effective, efficient, equitable, and safe."

There have been notable efforts to define the quality of specific health care services. One landmark effort to define quality reproductive health care services emerged in 1990. The Bruce-Jain definition of quality reproductive health care includes six elements focused on quality family planning care: choice of contraceptive methods; information given to patients; technical competence; interpersonal relationships; continuity and follow-up; and the appropriate constellation of services. ${ }^{6}$ Importantly, the framework does not identify minimum or optimum standards for quality care, but instead details areas of service delivery on which to focus. ${ }^{7}$ Since its initial 
development, scholars and major public health bodies have made modifications to the well-regarded framework by including other types of reproductive health services, ${ }^{8} 9$ and addressing access to services ${ }^{10}$ and health care structures, among other issues. ${ }^{11}$

In this paper, we focus on quality abortion care. We focus on abortion care because it remains unclear what constitutes quality abortion care ${ }^{12}$ and because improving the quality of abortion care may lead to reductions in community-level abortion stigma ${ }^{13}$ and abortion-related morbidities and mortality. ${ }^{14-18}$ Improving abortion quality may also lead to increased post-abortion contraceptive knowledge ${ }^{18}$ and uptake. ${ }^{19}$

Quality improvement efforts rely on the measurement and tracking of standardised indicators that provide insight into whose health care needs remain unmet, if there are any improvements needed in how health care is delivered in a facility, what health system issues are in need of change, and what legal and policy reforms are necessary. ${ }^{12}$ Reporting on these issues using standardised indicators has the potential to help drive quality improvements and influence the extent to which quality improvement efforts are supported by policymakers, philanthropic organisations, researchers, advocates, programme planners, and health care practitioners. ${ }^{20} 21$

Comprehensive quality improvement efforts track indicators at the structure, process and outcome levels. Indicators related to structure focus on the setting in which care occurs. Process indicators focus on what is done on the ground to give and receive care. Outputs are the results of process. Outcome indicators focus on what happens after care and how it affects health status. ${ }^{22}$

We aim to review indicators relevant to quality abortion care that address structure, process, outputs and outcomes to determine which indicators are most commonly used when assessing quality. To our knowledge, no such review exists. We see this review as critical, as it will help to determine if a common set of indicators is relied upon to measure quality abortion care, which is an essential step for identifying gaps in indicators of quality abortion care and ultimately establishing an agreed upon approach to measuring quality across a range of service delivery settings and contexts. This will help to ensure that efforts to measure and improve abortion care quality are informed and coordinated. ${ }^{23}$

\section{METHODS}

From January through March 2015, we conducted a search for peer-reviewed and grey literature that put forth newly developed indicators of quality abortion care. Specifically, we first searched the following scientific databases: Google Scholar, PubMed and Web of Science. We used combinations of the following search terms: 'quality', 'abortion' and 'indicators'. We excluded papers not available in English and excluded papers focused on non-human research and on people 65 years of age or older. We included all papers published before January 2015. We identified 44 relevant publications for review. Many of these publications were evaluations of existing indicators. To avoid duplicating indicators already identified in our review, we did not include these articles unless they noted that they had modified the indicator or operationalised an indicator in a new way. This left 13 peer-reviewed papers for review. We supplemented this search and looked for grey literature on various internet search engines, such as Google, using the same search terms. This resulted in eight additional relevant reports.

One author reviewed all studies and reports and recorded information about indicators in an Excel data collection sheet, listing the name of the indicator, the definition (if provided), the study type from which the indicator came, the country of the study, and the source. The study team then reviewed the list of indicators and collapsed duplicate or similar indicators. Next, we organised the indicators by whether they were related to structure, process, output or outcome. After reviewing the indicator definitions, we grouped similar indicators together and created subthemes. We then noted where indicators had no common agreement to date in the literature (cited by one paper), moderate agreement (cited by two or three papers) or a high level of agreement (cited by four or more papers). Because there is no gold standard for how many times an indicator needs to be cited for it to be considered well accepted or agreed upon, we defined levels of agreement after finding - as described below - how few indicators were cited more than once.

\section{RESULTS}

In total, we reviewed 21 peer-reviewed and grey papers published between 1991 and 2014. Indicators reported in these papers were derived through various methods. Almost half of the indicators (48\%) were developed through the process of creating abortionfocused public health frameworks, monitoring plans, or treaties. Some indicators were created after interviewing or surveying abortion clients (33\%) or after interviewing or surveying individuals with expertise in abortion care (14\%). Least commonly, indicators were developed as a byproduct of creating best practices for providing abortion (10\%). ${ }^{\mathrm{i}}$ Over half of the reviewed papers focused on global indicators of quality. The few papers with a country- or region-specific lens were focused in the USA (14\%), Africa or Sub-Saharan Africa (10\%), Finland (5\%), Mexico (5\%), Nepal (5\%), Sweden (5\%) and Vietnam (5\%).

${ }^{\mathrm{i} O n e}$ paper was counted twice: it developed indicators after interviewing both abortion clients and experts in abortion care. 
Our initial list included 207 indicators. After similar and duplicative indicators had been combined, 75 unique indicators of quality abortion care remained. The majority of indicators were cited by only one paper (range 1-9 citations) (Table 1). Indeed, there was little agreement in the literature for $56 \%$ of indicators, some agreement for $27 \%$ of indicators, and a high level of agreement for $17 \%$ of indicators.

\section{Structure indicators}

Almost one-third of the indicators focus on assessing the structures necessary to provide high-quality abortion care (Table 2). The biggest subtheme within structure indicators and across all indicators was infrastructure. Twenty-eight percent of indicators overall fell into the infrastructure subtheme (Table 2). These indicators measure whether comprehensive communications, health, referral and transportations systems are in place alongside adequate health facilities, technologies, equipment, supplies and staff for highquality abortion care to be delivered (Table 3). The stated assumption behind these indicators is that, with such structures in place, people seeking abortion will be able to readily access affordable, geographically proximate, timely and integrated care. Three infrastructure indicators had high levels of agreement. The first of these, determining if there is an adequate number of staff knowledgeable about and trained in abortion care, was the only indicator we identified that had nine citations. The other two indicators, which focus on evaluating if timely services are available and if facilities are accessible, had four citations.

Three percent of indicators overall focus on the subtheme of laws and policies (Table 2). Within the law and policy subtheme, one indicator, which assesses if supportive laws and policies are in place, had a high level of agreement (Table 3).

\section{Process indicators}

Nearly half of the indicators focus on evaluating the processes that must be in place to provide high-quality

Table 1 Number of indicators with a given number of citations *

\begin{tabular}{ll}
\hline Frequency of citations & $\begin{array}{l}\text { Indicators with this } \\
\text { number of citations } \\
\mathbf{n}(\%)\end{array}$ \\
\hline 1 & $42(56)$ \\
2 & $14(19)$ \\
3 & $6(8)$ \\
4 & $5(7)$ \\
5 & $4(5)$ \\
6 & $1(1)$ \\
7 & $1(1)$ \\
8 & $1(1)$ \\
9 & $1(1)$ \\
\hline
\end{tabular}

*Percentages may not add up to 100 because of rounding.
Table 2 Indicator category and subthemes of abortion quality*

\begin{tabular}{lcc}
\hline Indicator category or subtheme & $\mathbf{n}(\%)$ \\
\hline Structure indicators & $23(31)$ \\
Infrastructure & $21(28)$ \\
Laws and policies & $2 \quad(3)$ \\
Process indicators & $34(45)$ \\
Technical competence & $12(16)$ \\
Client-provider interactions & $8(11)$ \\
Decision making & 5 & $(7)$ \\
Information provision & 4 & $(5)$ \\
Ancillary services & 3 & $(4)$ \\
Support & 2 & $(3)$ \\
Outputs & 4 & $(5)$ \\
Procedures provided & 4 & $(5)$ \\
Outcomes & $14(19)$ \\
Client and community knowledge & 5 & $(7)$ \\
Client and community attitudes & 5 & $(7)$ \\
Demographic trends in abortion & 2 & $(3)$ \\
Client morbidity and mortality & 2 & $(3)$ \\
\hline *Percentages may not add up to 100 because of rounding. &
\end{tabular}

abortion care (Table 2). The bulk of these indicators assess technical competence and make up $16 \%$ of the overall indicators (Table 2). More specifically, these indicators appraise the technical steps assumed necessary for delivering quality abortion care from the point of screening a client for the procedure, to performing an abortion, and to providing aftercare (Table 3). Although none of the technical competency indicators had a high level of agreement, one indicator - evaluating if appropriate pain management is provided - came close to the high level of agreement benchmark and had three citations.

Client-provider interactions make up the next subtheme of process indicators, representing $11 \%$ of indicators overall (Table 2). These indicators measure how staff at abortion facilities interact with abortion clients and assess if staff are treating women with dignity, withholding judgement, and providing confidential care (Table 3). Among the client-provider indicators, two had a high level of agreement: determining if respectful care is offered and if privacy is provided during an appointment both had five citations.

Next, 7\% of overall indicators focus on gauging decision making (Table 2) and assessing if women make informed decisions to terminate a pregnancy, which was commonly defined as a decision that is free of coercion and is documented (Table 3). One indicator within the decision-making subtheme, evaluating if counselling has been provided before the procedure, had a high level of agreement, with five citations.

Five percent of overall indicators focus on measuring if clients have been given abortion-relevant information in an appropriate format before the procedure (Table 2). There is a very high level of agreement, 
Table 3 Quality abortion care indicators

\begin{tabular}{|c|c|c|}
\hline Indicator category or subtheme & Indicator & References \\
\hline \multicolumn{3}{|l|}{ Structure indicators } \\
\hline 1) Infrastructure & $\begin{array}{l}\text { There are an adequate number of knowledgeable and trained staff available to provide } \\
\text { abortion care. }\end{array}$ & $7,12,24-30$ \\
\hline 2) Infrastructure & Timely services are available. & $24,27,31,32$ \\
\hline 3) Infrastructure & Facilities are geographically accessible. & $12,26,29,30$ \\
\hline 4) Infrastructure & Efficient, high-quality referral systems are in place. & 7, 33, 34 \\
\hline 5) Infrastructure & $\begin{array}{l}\text { Essential equipment, supplies and medications are available in sufficient quantity to address } \\
\text { needs. }\end{array}$ & $7,25,27$ \\
\hline 6) Infrastructure & Abortion care is provided in a clean facility. & 24,31 \\
\hline 7) Infrastructure & Abortion is provided in a facility with space for privacy. & 24,35 \\
\hline 8) Infrastructure & Abortion is provided in an emotionally and physically comforting space. & 28 \\
\hline 9) Infrastructure & Well-functioning systems for transportation and communications for abortion are in place. & 7 \\
\hline 10) Infrastructure & $\begin{array}{l}\text { Throughout the health system, there are mechanisms to review abortion-related } \\
\text { complications and deaths. }\end{array}$ & 7 \\
\hline 11) Infrastructure & $\begin{array}{l}\text { Women enter the health care system through decentralised service delivery points and } \\
\text { receive care at the lowest appropriate level of the networked system. }\end{array}$ & 7 \\
\hline 12) Infrastructure & $\begin{array}{l}\text { Abortion care services are integrated with or linked to the fullest available array of medical } \\
\text { and reproductive health services. }\end{array}$ & 7 \\
\hline 13) Infrastructure & $\begin{array}{l}\text { Services are effectively managed and administrative and logistical factors are not obstacles to } \\
\text { the timely delivery of high-quality care. }\end{array}$ & 7 \\
\hline 14) Infrastructure & $\begin{array}{l}\text { Fees for abortion services are within reach of women's ability to pay; emergency care is } \\
\text { provided regardless of women's ability to pay. }\end{array}$ & 7 \\
\hline 15) Infrastructure & $\begin{array}{l}\text { There is an adequate percentage of abortion care service sites that meet a defined standard } \\
\text { of quality. }\end{array}$ & 12 \\
\hline 16) Infrastructure & $\begin{array}{l}\text { Essential equipment, supplies and medication should be managed through a system of } \\
\text { inventory control, resupply and maintenance. }\end{array}$ & 7 \\
\hline 17) Infrastructure & Abortion technology is consistent with relevant regulatory requirements. & 7 \\
\hline 18) Infrastructure & Abortion technology is appropriate to specific service delivery settings. & 7 \\
\hline 19) Infrastructure & Abortion technology is manufactured to high standards. & 7 \\
\hline 20) Infrastructure & Abortion technology is acceptable to women and providers. & 7 \\
\hline 21) Infrastructure & Staff at abortion facilities have adequate supervision. & 7 \\
\hline 22) Law and policies & Abortion care must be accessible and not limited by administrative or policy barriers. & $7,12,29,36$ \\
\hline 23) Law and policies & $\begin{array}{l}\text { Regulations, guidelines and other policy documents have been developed, approved by } \\
\text { national/sub-national governments, and/or disseminated to health care facilities that are } \\
\text { supportive of access to safe abortion care consistent with WHO guidance. }\end{array}$ & 25 \\
\hline \multicolumn{3}{|l|}{ Process indicators } \\
\hline 24) Technical competence & Appropriate pain management techniques are in place. & $24,28,37$ \\
\hline 25) Technical competence & Clients are screened for requested procedures. & 27,33 \\
\hline 26) Technical competence & Clinical histories are taken. & 27,33 \\
\hline 27) Technical competence & $\begin{array}{l}\text { Physical assessments of general and sexual and reproductive health are performed (including } \\
\text { confirmation of gestational age). }\end{array}$ & 27,33 \\
\hline 28) Technical competence & $\begin{array}{l}\text { Staff follow approved guidelines and protocols for medical, surgical, and incomplete } \\
\text { abortion. }\end{array}$ & 27,33 \\
\hline 29) Technical competence & Staff use appropriate technologies. & 33,38 \\
\hline 30) Technical competence & $\begin{array}{l}\text { Follow-up care is provided, where women's experience with abortion and pregnancy status } \\
\text { are assessed. }\end{array}$ & 33,34 \\
\hline 31) Technical competence & Clients are asked the reason for the visit. & 33 \\
\hline 32) Technical competence & If the woman aborts at the clinic, products of conception are examined to confirm expulsion. & 33 \\
\hline 33) Technical competence & Appropriate infection prevention protocols are in place. & 27 \\
\hline 34) Technical competence & Best practice guidelines are followed for monitoring during recovery period. & 27 \\
\hline 35) Technical competence & Staff only use techniques for which they are adequately trained. & 27 \\
\hline 36) Client-provider interactions & Staff offer respectful care. & $7,27,31,33,35$ \\
\hline 37) Client-provider interactions & Staff work to ensure privacy during the visit. & $27,31-33,39$ \\
\hline 38) Client-provider interactions & Staff have positive interactions with clients. & $24,28,39$ \\
\hline 39) Client-provider interactions & Staff provide confidential care. & 7, 24, 39 \\
\hline 40) Client-provider interactions & Staff should promote client's dignity. & 27,32 \\
\hline 41) Client-provider interactions & Care is tailored to women's individual circumstances and needs. & 39 \\
\hline
\end{tabular}


Table 3 Continued

\begin{tabular}{l} 
Indicator category or subthe \\
\hline 42) Client-provider interactions \\
43) Client-provider interactions \\
44) Decision making \\
45) Decision making \\
46) Decision making \\
47) Decision making \\
48) Decision making \\
49) Information provision
\end{tabular}

50) Information provision

51) Information provision

52) Information provision

53) Ancillary services

54) Ancillary services

55) Ancillary services

56) Support

57) Support

Output indicators

58) Procedures provided

59) Procedures provided

60) Procedures provided

61) Procedures provided

Outcome indicators

62) Client and community knowledge

63) Client and community knowledge

64) Client and community knowledge

65) Client and community knowledge

66) Client and community knowledge

67) Client and community attitudes

68) Client and community attitudes

69) Client and community attitudes

70) Client and community attitudes

71) Client and community attitudes

72) Client behaviours

73) Client behaviours

74) Client morbidity and mortality

75) Client morbidity and mortality

\section{Indicator}

Staff hold non-judgemental attitudes.

Staff-client interactions promote an atmosphere of trust.

Clients are provided high-quality, supportive counseling.

Staff obtain informed consent from clients.

Clients are provided the opportunity to explore views on abortion options and methods.

Staff trust clients' abilities to make informed decisions.

Provider-client interactions are absent of provider bias or coercion.

Staff explain all aspects of abortion care to clients (current condition, treatment plan,

follow-up needs, and potential post-abortion complications and how to obtain appropriate post-abortion care).

Staff provide clients the opportunity to express concerns, ask questions, and receive accurate, understandable answers.

Staff provide basic information about conception, pregnancy and pregnancy options.

Staff use age-appropriate, non-clinical language.

Staff directly provide or offer referrals for a range of sexual and reproductive health services, including contraception and screening and treatment for HIV and STIs.

Staff provide information about a range of sexual and reproductive health needs including: contraception, fertility, gender-based violence, prenatal care, cancer screening, and screening and treatment for HIV and STIs.

Abortion care is no way contingent on prior acceptance of contraception.

References

If desired, staff ensure emotional comfort provided to client from support person during abortion.

Staff explore what kind of support client has for their decision.

$27,28,34,35$

27,33

7,33

7

7, 27, 31-33, 35, 39

$7,27,33,35$

33

33

$7,12,25,27,29,33$, 38,39

$7,27,30,32,33,39$

7

$24,28,33$

33

Assess the number of safe induced abortion procedures.

Assess the proportion of procedures that are induced.

Assess the percentage of uterine evacuations performed with appropriate technologies.

Assess the percentage of abortion procedures performed with preferred technologies.

The community is aware of the availability of safe abortion.

Clients understand the information given during clinic visits.

An adequate number or percentage of women of reproductive age have accurate knowledge of abortion laws.

An adequate number or percentage of women of reproductive age can identify a nearby source of safe abortion care.

Women are aware of the full range of services available to them throughout the health care system.

Clients are satisfied with abortion care.

$12,30,40$

29

29

12

26,27

35

12

12

There is an adequate number or percentage of women with positive attitudes toward seeking abortion care.

Women perceive few financial, geographic and cultural barriers to safe abortion care.

Clients feel that the staff have a high level of clinical competency.

Clients have confidence and trust in staff providing care.

Assess overall abortion-seeking behaviours and trends.

Assess the percentage of abortions that occur at or before 10 weeks' gestations.

There is a low number of admissions for treatment of abortion complications.

There is a low percentage of maternal deaths as a result of abortion.

\section{7}

STI, sexually transmitted infection; WHO, World Health Organization.

with seven citations, that an assessment should be conducted about whether clients have been provided information about all aspects of abortion care, including their current condition, treatment plan, follow-up needs, and potential post-abortion complications and how to obtain appropriate post-abortion care (Table 3). It is also commonly agreed that it should be determined if clients are provided the opportunity to express concerns, ask questions, and receive accurate, understandable answers about their abortion care.

Another $4 \%$ of overall indicators focus on ancillary services (Table 2). An underlying assumption of these indicators is that ancillary services are a 
critical component of quality abortion care. To determine if quality ancillary services are provided, indicators evaluate if women receive information and referrals about ancillary services such as contraception, gender-based violence, pregnancy and sexually transmitted infections (STIs), or if women are directly provided these services at the time of their abortion (Table 3). There was a high level of agreement about the need to assess if information and referrals for ancillary services are offered or if ancillary services are directly provided, with these indicators being among the top five most frequently cited indicators.

Last in the process indicators, $3 \%$ of all indicators focus on external emotional support and measuring if women have support from someone in their lives during their abortion decision and the procedure itself (Table 2). None of the support indicators had a high level of agreement (Table 3). However, one indicator in this subtheme - evaluating whether women have emotional care from a support person during their abortion procedure - had a moderate level of agreement.

\section{Output indicators}

Five percent of indicators focus on the outputs that signify that high-quality abortion care has been provided (Table 2). There is only one subtheme within the output indicators, focused on outputs of the abortion procedure itself. These indicators measure the number of safe abortions performed and the number of abortions performed with appropriate, acceptable technologies (Table 3). None of the output indicators had a high level of agreement, although one, tracking the number of safe abortions performed, reached a moderate level of agreement.

\section{Outcome indicators}

Twenty percent of overall indicators focus on the outcomes that occur when quality abortion care is provided. One subtheme among the outcome indicators is focused on tracking client and community knowledge about abortion, and made up 7\% of overall indicators (Table 2). These indicators assess clients' and communities' knowledge about abortion laws, regulations and services, as well as other health services (Table 3). None of these indicators reached a high level of agreement and only one indicator in the knowledge subtheme - determining if the community is aware of the availability of abortion services - is cited more than once.

Another outcome subtheme, making up 7\% of overall indicators, is measurement of client and community attitudes towards abortion care. Underlying these indicators is the assumption that after quality abortion care is provided, clients and the community feel positively about abortion care and the staff providing that care, and that there are few perceived barriers to abortion care. None of the indicators in the attitudes' subtheme had a high level of agreement, and only one indicator in this area - checking if clients are satisfied with abortion care - was cited more than once.

A third subtheme is client behaviours. The two indicators in this subtheme are focused on measuring the number of abortions sought overall and the number of abortions provided at or before 10 weeks' gestation. It is not explicitly stated in what direction these numbers will move, although we infer that the underlying assumption is that, after quality abortion care has been provided, the number of overall abortions sought will decline and the number of abortions that occur at or before 10 weeks' gestation will increase, presumably due to ancillary services provided during abortion care. Neither of the indicators within this subtheme was cited more than once.

Last, 3\% of overall indicators focus on abortionrelated morbidities and mortality (Table 2), and assume that, when quality abortion care is provided, abortion-related morbidities and mortalities will decline, and hence should be tracked over time (Table 3). There is a high level of agreement for both of the indicators within this subtheme.

\section{DISCUSSION}

In this review, we found that many qualityof-abortion-care indicators have been put forth in the peer-reviewed and grey literature, and that there is inconsistency in how quality abortion care has been measured and tracked. This suggests that there is little agreement about what constitutes quality abortion care. At the same time, the diversity of indicators reveals that abortion care quality is a broad and multifaceted issue, spanning sectors such as policy and health systems, while also addressing women's decision making and emotional supports. In many ways, the diversity of indicators represent progress, as scholars have noted that quality abortion care has historically been associated with the technical skill to perform abortion and the lack of abortion-related complications, ${ }^{35}$ and that less attention has been paid to the sociocultural aspects of the health care, such as good communication between providers and clients appropriate levels of privacy, and the receipt of high-quality information. $^{15} 35$ This approach to abortion care quality is consistent with global efforts to define quality health services as constituting much more than what happens in an examination room. ${ }^{3}$

At the same time, some common themes did emerge in our cataloguing of indicators. We found that existing indicators for abortion care quality are largely focused on evaluating the process of providing care and assessing the structures that are in place to offer care. Less commonly, indicators address measuring what happens after care is provided - how outputs and outcomes are affected by the relative quality of abortion care provided. 
We also note that there was only a high level of agreement for $17 \%$ of the identified indicators. Moving from the most to the least commonly cited indicator for abortion care quality, the indicators for which there was a high level of agreement were assessing (1) the availability of trained staff to provide care, (2) whether referrals for, or direct provision of, a range of sexual and reproductive health services are offered, (3) if all aspects of abortion care are explained to women, (4) if information about a range of sexual and reproductive health services is offered, (5) whether staff offer respectful care, (6) if staff work to ensure privacy, (7) if high-quality, supportive counselling is provided, (8) the abortion complication rate, (9) if services are offered in a timely manner, (10) if services are geographically accessible, (11) whether policies support access to abortion, (12) if women have the opportunity to express concerns, ask questions, and receive answers, and (13) the rate of maternal deaths due to abortion complications.

We consider why these indicators rose to the top in our review. We speculate that the attention paid to the importance of assessing if there is an adequate number of well-trained staff is related to the welldocumented, inadequate supply of abortion providers worldwide, which can impede women's access to the service, and to the fact that quality and functionality of any health care delivery system depends on the availability of medical personnel. ${ }^{42}{ }^{43}$ In relation to this, the necessity of determining if women can access care emerges in indicators 9-11. Also, several indicators $(3,5,6$ and 7$)$ are thematically focused on measuring if women have been treated with dignity and if they have been supported in making informed decisions. The fact that two of the top 13 indicators evaluate ancillary services suggests that many consider abortion to be only one part of women's broader reproductive life plans and that addressing only one aspect of that plan is insufficient. Last, we suggest that the reason that assessing the rate of abortion-related morbidity and mortality shows up relatively low in the list is the difficulty of measuring health care outcomes (since they often occur after point of service), and not a lack of interest in abortion-related health outcomes. Indeed, prior scholars have pointed to the importance of capturing process indicators that ultimately influence health outcomes, instead of just focusing on difficult-to-capture health outcomes. ${ }^{21}$

We see the fact that many indicators had no or moderate agreement as a cue that there is a need to improve the current indicators used to measure quality of care in order to ensure these indicators are responsive to the priorities and needs of women and their service providers. A more streamlined list of indicators must be developed. A streamlined list of indicators would help those who provide abortion care services to focus on improving aspects of care that have the highest potential to transform quality.
The challenge of selecting an efficient list of appropriate indicators to track and improve quality is not unique to abortion care. Indeed, there is no shortage of frameworks for determining what constitutes quality health care across many different medical fields. ${ }^{44-49}$

Building on these frameworks for selecting key quality indicators for other health care services, we suggest the following steps for doing so for abortion care. First, as is best practice when developing quality indicators, ${ }^{23} 50$ it would be beneficial to receive additional feedback from women seeking abortions about the appropriateness of current abortion care quality indicators by asking them to interrogate if the indicators address what is most important to them when they are seeking and obtaining abortion care services. It would also be beneficial to have similar discussions about proposed quality indicators with providers offering care. Gathering this feedback would help in selecting and prioritising indicators that have the most relevance to those in need of abortion and those delivering the service. Lessons about the importance of developing indicators that appreciate end users' needs and preferences have already been learned in the field of family planning. ${ }^{20}$

Next, we suggest that the quality of evidence used to develop indicators for abortion care quality must be assessed. Such assessments are considered best practice when developing indicators to measure quality of care $^{21}$ and have been conducted for other reproductive health care services. Indeed, when the Center for Disease Control and Prevention (CDC) and the Office of Population Affairs (OPA) in the USA worked to determine indicators of quality family planning, they included an assessment of the quality of evidence about recommendations to provide quality family planning, the consequences of implementing the recommendations, and the relative importance of those consequences. ${ }^{44}$ Also, given the diversity of service delivery contexts for abortion across the globe, we also suggest a need to assess which indicators work well across multiple settings and which indicators are best used in specific country or regional contexts.

After taking the above steps, indicators should be assessed for the feasibility of collecting data on the indicator, the validity of current measures for collecting those data, and the ethical appropriateness of collecting the data. 32128

Our hope is that these steps would lead to a lean hierarchy of indicators for quality abortion care, a necessary step to ensure that efforts to measure and improve abortion care quality are informed and coordinated. ${ }^{23}$ Sitting at the top of that hierarchy would be a streamlined set of indicators that are evidencebased, impactful, consistently prioritised by those seeking abortion and those providing it, and appropriate to collect. These indicators would represent 'must haves' in abortion care. Next in the hierarchy would 
be indicators that are new concepts, have been less rigorously tested, have inconsistent support from those seeking abortion and those providing it, and are intensive or difficult to collect. These indicators would represent quality areas still to be explored or those that would be 'nice to haves' in abortion care.

\section{Strengths and limitations}

We aimed to develop a comprehensive catalogue of abortion care quality indicators. A strength of our approach is our review of both peer-reviewed and grey literature. However, it is possible that not all indicators were captured. In addition, it is possible that, when indicators were collapsed, important nuances about quality abortion care were lost. We were conservative in our approach to collapsing indicators in efforts to mitigate this possibility.

\section{Conclusion}

Efforts to improve the quality of abortion care rely heavily on the availability and use of concrete quality indicators. The identified catalogue of abortion care quality indicators addresses the multiple dimensions that need to be assessed to monitor and improve this quality. However, the current catalogue of indicators is also cumbersome, lacking alignment, and relatively untested. This highlights the need to develop a streamlined set of indicators that are evidence-based, impactful, consistently prioritised by those seeking abortion and those providing it, and feasible to collect. Doing so would assist in improving the quality of abortion care, which is necessary to improve women's experiences with care, reduce abortionrelated morbidities and mortalities, and reduce any disparities in these outcome areas.

Acknowledgements The authors thank Emily Melnick for her assistance reviewing the literature.

Funding The Packard Foundation provided financial support for this work; views and opinions expressed in this paper are those of the authors and do not necessarily represent the views of the Packard Foundation.

Competing interests None declared.

Provenance and peer review Not commissioned; externally peer reviewed.

Data sharing statement As this is a literature review, all data are publicly available.

\section{REFERENCES}

1 World Health Organization. Quality of Care: A Process for Making Strategic Choices in Health Systems. Geneva: World Health Organization, 2006 [cited 20 November 2015]. http:// www.who.int/management/quality/assurance/QualityCare_B. Def.pdf

2 World Health Organization. Everybody's Business: Strengthening Health Systems to Improve Health Outcomes: WHO's Framework for Action. Geneva: World Health Organization, 2007 [cited 20 November 2015]. http://www. who.int/healthsystems/strategy/everybodys_business.pdf

3 Creel LC, Sass JV, Yinger NV. Overview of Quality of Care in Reproductive Health: Definitions and Measurements of Quality
-Eldis. Washington DC: Population Reference Bureau, 2002 [cited 20 November 2015]. http://www.eldis.org/go/ home\&id=24664\&type $=$ Document\#.Vk9tueJBG_E

4 National Committee for Quality Assurance. The Essential Guide to Health Care Quality. Washington DC: National Committee for Quality Assurance, 2014 [cited 20 November 2015]. http://www.ncqa.org/Portals/0/Publications/Resource\% 20Library/NCQA_Primer_web.pdf

5 Institute of Medicine. Crossing the Quality Chasm: The IOM Health Care Quality Initiative. Institute of Medicine, 2013 [cited 20 November 2015]. http://iom.nationalacademies.org/ Global/News\%20Announcements/Crossing-the-QualityChasm-The-IOM-Health-Care-Quality-Initiative.aspx

6 Bruce J. Fundamental elements of the quality of care: a simple framework. Stud Fam Plann 1990;21:61-91.

7 Leonard AH, Winkler J. A Quality of Care Framework for Abortion Care. Carrboro, NC: IPAS, 1991 [cited 20 November 2015]. http://www.ncbi.nlm.nih.gov/nlmcatalog/101052755

8 Mora G, Betts C, Gay J, et al. Quality of Care in Women's Reproductive Health: A Framework for Latin America and the Caribbean. Washington DC: Pan American Health Organization, 1993 [cited 20 November 2015]. http://www. popline.org/node/340462

9 World Health Organizaiton. Reproductive Health Indicators for Global Monitoring: Report of the Second Interagency Meeting. Geneva: World Health Organization; [cited 20 November 2016]. http://www.who.int/reproductivehealth/publications/ monitoring/RHR_01_19/en/

10 Bertrand JT, Hardee K, Magnani RJ, et al. Access, quality of care and medical barriers in family planning programs. Int Fam Plan Perspect 1995;21:64.

11 AbouZahr C, Vlassoff C, Kumar A. Quality health care for women: a global challenge. Health Care Women Int 1996;17:449-467.

12 Benson J. Evaluating abortion-care programs: old challenges, new directions. Stud Fam Plann 2005;36:189-202.

13 Jewkes RK, Gumede T, Westaway MS, et al. Why are women still aborting outside designated facilities in metropolitan South Africa? BJOG 2005;112:1236-1242.

14 Chowdhury ME, Botlero R, Koblinsky M, et al. Determinants of reduction in maternal mortality in Matlab, Bangladesh: a 30-year cohort study. Lancet 2007;370:1320-1328.

15 Jewkes R, Rees H. Dramatic decline in abortion mortality due to the Choice on Termination of Pregnancy Act. S Afr Med J 2005;95:250.

16 Hord C, David HP, Donnay F, et al. Reproductive health in Romania: reversing the Ceausescu legacy. Stud Fam Plann 1991;22:231-240.

17 Billings DL, Fuentes Velásquez J, Pérez-Cuevas R. Comparing the quality of three models of postabortion care in public hospitals in Mexico City. Int Fam Plan Perspect 2003;29:112-120.

18 Bhandari A, Mo Hom N, Rashid S, et al. Experiences of abortion in Nepal and menstrual regulation in Bangladesh: a gender analysis. Gender Dev 2008;16:257-272.

19 Tabbutt-Henry J, Graff K. Client-provider communication in postabortion care. Int Fam Plan Perspect 2003;29:126-129.

20 Dehlendorf C, Bellanca H, Policar M. Performance measures for contraceptive care: what are we actually trying to measure? Contraception 2015;91:433-437.

21 Blanchard K, B Elul, S RamaRao. Reproductive Health Indicators: Moving Forward. New York: Population Council, 1999. 
22 Donabedian A. The quality of care: how can it be assessed? JAMA 1988;260:1743-1748.

23 US Department of Health and Human Services. National Strategy for Quality Improvement in Health Care: 2014 Annual Progress Report to Congress. Washington DC: US Department of Health and Human Services, 2014 [cited 20 November 2015]. http://www.ahrq.gov/workingforquality/ reports/annual-reports/nqs2014annlrpt.pdf.

24 McLemore MR, Desai S, Freedman L, et al. Women know best--findings from a thematic analysis of 5,214 surveys of abortion care experience. Women Health Iss 2014;24:594-599.

25 Seims S, Khadduri R. Measuring improvements in sexual and reproductive health and rights in sub-Saharan Africa. Reprod Health Matters 2012;20:177-187.

26 The African Union Comission. General Comment No. 2 on Article 14.1 (a), (b), (c) and (f) and Article 14. 2 (a) and (c) of the Protocol to the African Charter on Human and Peoples' Rights on the Rights of Women in Africa/Legal Instruments/ ACHPR. 2010 [cited 16 February 2016]. http://www.achpr.org/ instruments/general-comment-two-rights-women/

27 International Planned Parenthood Federation. First Trimester Abortion Guidelines and Protocols: Surgical and Medical Procedure. International Planned Parenthood Federation, 2010 [cited 16 February 2016]. http://www.ippf.org/sites/default/files/ abortion_guidelines_and_protocol_english.pdf

28 Regmi K, Madison J. Ensuring patient satisfaction with second-trimester abortion in resource-poor settings. Int $J$ Gynaecol Obstet 2010;108:44-47.

29 Healy J, Otsea K, Benson J. Counting abortions so that abortion counts: Indicators for monitoring the availability and use of abortion care services. Int J Gynaecol Obstet 2006;95:209-220.

30 Bulatao RA, Ross JA. Rating maternal and neonatal health services in developing countries. Bull World Health Organ 2002;80:721-727.

31 Becker D, Díaz-Olavarrieta C, Juárez C, et al. Clients' perceptions of the quality of care in Mexico city's public-sector legal abortion program. Int Perspect Sex Reprod Health 2011;37:191-201.

32 The Picker Institute. From the Patient's Perspective: Quality of Abortion Care. The Picker Institute, 1999 [cited 16 February 2016]. https://kaiserfamilyfoundation.files.wordpress.com/ 1999/04/ppqofabortioncare.pdf

33 Ipas. Woman-Centered, Comprehensive Abortion Care Trainer's Manual. 2nd edn. Ipas, 2014 [cited 16 February 2016]. http:// www.ipas.org/en/Resources/Ipas\%20Publications/ WomanCentered-Comprehensive-Abortion-Care-TrainersManual-Second-Edition.aspx

34 Sihvo S, Hemminki E, Kosunen E, et al. Quality of care in abortion services in Finland. Acta Obstet Gynecol Scand 1998;77:210-217.

35 Nguyen MHN, Gammeltoft T, Rasch V. Situation analysis of quality of abortion care in the main maternity hospital in Hai Phòng, Viet Nam. Reprod Health Matters 2007;15:172-182.
36 United Nations. Report on the International Conference on Population and Development. New York: United Nations, 1995 [cited 16 February 2016]. https://www.unfpa.org/sites/default/ files/event-pdf/icpd_eng_2.pdf

37 Taylor D, Postlethwaite D, Desai S, et al. Multiple determinants of the abortion care experience: from the patient's perspective. Am J Med Qual 2013;28:510-518.

38 Ipas. Medical Abortion Training Guide. Chapel Hill, NC: Ipas, 2010.

39 Hyman A, Kumar A. A woman-centered model for comprehensive abortion care. Int J Gynaecol Obstet 2004;86:409-410.

40 Dixon-Mueller R, Germain A. Fertility regulation and reproductive health in the millennium development goals: the search for a perfect indicator. Am J Public Health 2007;97:45-51.

41 Swedish Association of Local Authorities and Regions and Swedish National Board of Health and Welfare. Quality and Efficiency in Swedish Health Care-Regional Comparisons 2012. Stockholm: The National Board of Health and Welfare, 2012 [cited 16 February 2016]. https://www.socialstyrelsen.se/ Lists/Artikelkatalog/Attachments/19072/2013-5-7.pdf

42 Henshaw SK. Factors hindering access to abortion services. Fam Plann Perspect 1995;27:54-59, 87.

43 Agency for Healthcare Research and Quality. 2010 National Healthcare Quality and Disparities Report. Washington DC: US Department of Health and Human Services, 2011. [cited 20 November 2015]. http://archive.ahrq.gov/research/findings/ nhqrdr/nhqrdr10/qrdr10.html

44 Gavin L, Moskosky S, Carter M, et al. Providing quality family planning services: Recommendations of CDC and the U.S. Office of Population Affairs. MMWR Recomm Rep 2014;63 (RR-04):1-54.

45 Campbell SM, Braspenning J, Hutchinson A, et al. Research methods used in developing and applying quality indicators in primary care. Qual Saf Health Care 2002;11:358-364.

46 Sibanda T, Fox R, Draycott TJ, et al. Intrapartum care quality indicators: a systematic approach for achieving consensus. Eur J Obstet Gyn R B 2013;166:23-29.

47 Earle CC, Park ER, Lai B, et al. Identifying potential indicators of the quality of end-of-life cancer care from administrative data. J Clin Oncol 2003;21:1133-1138.

48 Indicators of the quality of trauma care and the performance of trauma systems-Gruen-2011-British Journal of Surgery —Wiley Online Library [cited 2 February 2016]. http:// onlinelibrary.wiley.com/doi/10.1002/bjs.7754/full

49 Quality Indicators for Inflammatory Bowel Disease: Inflammatory Bowel Diseases. LWW. [cited 2 February 2016]. http://journals.lww.com/ibdjournal/Fulltext/2013/03000/ Quality_Indicators_for_Inflammatory_Bowel_Disease_.25.aspx

50 World Health Organization. Reproductive Health Indicators: Guidelines for their Generation, Interpretation and Analysis for Global Monitoring. Geneva: World Health Organization, 2006 [cited 20 November 2015]. http://apps.who.int/iris/bitstream/ 10665/43185/1/924156315X_eng.pdf 